\title{
Neuroprotection after infection-sensitized neonatal \\ hypoxic-ischemic brain injury
}

Hemmen Sabir,, Affl

Corresponding Affiliation: Aff1

Damjan Osredkar, $\stackrel{\text { Aff2 }}{,}$

Elke Maes,,$\stackrel{\text { Aff2 }}{,}$

Thomas Wood, $\stackrel{\text { Aff2 }}{,}$

Marianne Thoresen, $\underline{\text { Aff2 }}$

ArticleInfo

\begin{tabular}{|l|l|l|l|l||}
\hline \hline ArticleID & $:$ & 51 \\
\hline \hline ArticleDOI & $:$ & $10.1186 / 2194-7791-2-\mathrm{S} 1-\mathrm{A} 11$ \\
\hline \hline ArticleCitationID & $:$ & A11 \\
\hline \hline ArticleSequenceNumber & $:$ & 11 \\
\hline \hline ArticleCategory & $:$ & Meeting abstract \\
\hline \hline ArticleFirstPage & $:$ & 1 & \\
\hline \hline ArticleLastPage & $:$ & 2 \\
\hline \hline ArticleHistory & $:$ & $\begin{array}{l}\text { RegistrationDate } \\
\text { OnlineDate }\end{array}$ \\
\hline \hline ArticleCopyright & & $\begin{array}{l}\text { Sabir et al.2015 } \\
\text { This article is published under license to BioMed Central } \\
\text { Ltd. This is an Open Access article distributed under the } \\
\text { terms of the Creative Commons Attribution License } \\
\text { (lttp://creativecommons.org/licenses/by/4.0), which permits } \\
\text { unrestricted use, distribution, and reproduction in any } \\
\text { medium, provided the original work is properly cited. }\end{array}$ \\
\hline
\end{tabular}

Aff1

Department of General Pediatrics, Neonatology and Pediatric Cardiology, Children's Hospital, Heinrich-Heine University Düsseldorf, Düsseldorf, Germany

Aff2

Department of Physiology, Institute of Basic Medical Sciences, University of Oslo, Oslo, Norway

Abstracts of the 51 st Workshop for Pediatric Research

51st Workshop for Pediatric Research

Göttingen, Germany 
This supplement has not been sponsored.

Meeting abstracts

\section{Background}

Therapeutic hypothermia (HT) is standard treatment after perinatal hypoxic-ischemic (HI) injury. However, around $50 \%$ of asphyxiated newborns still suffer poor outcome, some of which may have been exposed to perinatal infection prior to HI, as infection may increase vulnerability to HI injury.

\section{Aims}

To develop an infection-sensitized newborn animal model of HI injury and to investigate whether HT is neuroprotective in this setting.

\section{Methods}

Seven day old rat pups were injected either with vehicle ( $\mathrm{NaCl} 0.9 \%)$ or E.coli lipopolysaccharide (LPS). Subsequently, they were exposed to left carotid ligation followed by global hypoxia inducing a unilateral HI injury. Pups were randomized to the following treatments: 1) Vehicle treated HI-pups receiving normothermia treatment (NT) (Veh-NT); 2) LPS treated HI-pups receiving NT treatment (LPS-NT); 3) vehicle treated HI-pups receiving HT treatment (Veh-HT); or 4) LPS treated HI-pups receiving HT treatment (LPS-HT). Relative area loss of the left/right hemisphere and the areas of hippocampi were measured after one-week survival.

\section{Results}

Mean brain area loss in the Veh-NT group was $11.2 \% \pm 14 \%$. The brain area loss in LPS-NT pups was $29.8 \% \pm 17 \%$, being significantly higher than in the Veh-NT group $(\mathrm{p}=0.002)$. The Veh-HT group had a significantly smaller brain area loss $(5.4 \% \pm 6 \%)$ when compared to Veh-NT group $(\mathrm{p}=0.043)$. The LPS-HT group showed a brain area loss of $32.5 \% \pm 16 \%$, being significantly higher than in the Veh-HT group $(\mathrm{p}<0.001)$. LPS-HT group also had significantly smaller size of the left hippocampus. LPS-sensitization significantly decreased the sizes of the right, unligated-hemispheres, independent of post-HI treatment.

\section{Conclusions}

Therapeutic hypothermia is not neuroprotective in this infection-sensitized HI brain injury model. Lack of neuroprotection was particularly seen in the hippocampus. The exact mechanisms remain unclear and further investigation is needed to explain why a neuroprotective therapy loses its efficacy and how hypothermia may be optimized. 